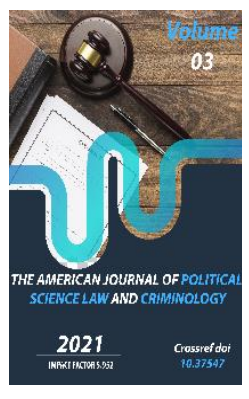

\title{
State Of Stress In Legal Situations
}

Makhmudova H. T.

PhD In Psychology, Associate Professor, Academy Of The Ministry Of Internal Affairs Of The Republic Of Uzbekistan, Expert Psychologist Of The Republican Center, Uzbekistan

Copyright: Original content from this work may be used under the terms of the creative commons attributes 4.0 licence.

\section{ABSTRACT}

The article discusses various scientific concepts of emotional states, considered as stress. This state in legally significant situations is related to situations in which control of the will and the ability to predict one's behavior are lost. The above circumstances are equated to the emotional states of a person in legally significant circumstances, when a person is provoked by circumstances exceeding the limit of his adaptive endurance.

\section{KEYWORDS}

Mental state, stress, frustration, anxiety, object of research, emotional experience.

\section{INTRODUCTION}

In numerous literary sources, the concept of concept. Moreover, the values can be divided "stress" is presented as a polysemantic according to several criteria, which include: 

a) A situational feature, where stress is viewed as events;
b) Sign - experiences, considering stress as a reaction;
c) A sign, where stress is considered as an intermediate variable.

In the context of our research, stress is viewed as a transactional process, i.e. as a process of human interaction with the outside world. R.S. Lazarus et al. Note that the transactional process begins with a specific assessment of an event and one's own coping resources, resulting in sometimes stressful emotions that have a legal significance determined by our research position [1].

If we consider events as stressors and systematize these stressors by the size of negative significance and by the time required for adaptation, then the object of our research interest can be stressors of critical life events, like the events of criminal incidents, or events related to the adoption of legal decisions in extreme situations. choice.

Many existing models of the complex interaction of various psychological factors influencing stressful experiences are taken into account in a combination of internal and external stress factors leading to unsuccessful adaptation. And in a criminal incident, this unsuccessful adaptation almost always leads to destructive actions that have legal consequences for the individual, the threshold of adaptive capabilities of which does not coincide with the requirements of the real environment

In literary sources, stress syndrome is given special importance, which is added to various conceptual provisions considering the phenomenology of the stress state. So, according to the provisions of M.I. Enikeeva, a stressful state inevitably occurs in all cases of a sudden appearance of a threat to life or to a person's social prestige. The author also attributes the cause of the stressful state, which is generated by systematic life failures. Considering the options for a person's stressful reaction to the harmfulness of the external or internal environment, the author identifies the possibilities of getting out of a stressful state in accordance with the adaptive capabilities of a person. According to L.A. Kitaeva-Smyk, the qualitative difference in human adaptation to stressful conditions is determined by the state of his life experience, as well as the innate qualities of the nervous system [2].

For all the danger of stress to life, according to the concept of G. Selje, "good stress" (mobilizing) allows to work out individual's adaptation mechanisms. However in the context of legally significant situations, adaptive mechanisms when mobilizing energy resources of a person in legally significant circumstances, acquire a different quality, and this quality has to do with destructive mobilization, eventually leading to actions of destructive nature.

In the formulation of V.F. Engalychev and S.S. Shipshin, mental tension (stress) is a condition arising at the person in an extreme (unusual, new or threatening) situation. And in this case, the impact of this state on the mental activity of a person is ambiguous and depends on the features of the stressful situation and the individual psychological qualities of the stressed person. On some people mental tension acts mobilizingly, others, on the contrary, feel its disorganizing influence [3].

According to the authors, the specificity of the state of mental tension differs from the state 
of affect, and these differences have to do primarily with the dynamics of occurrence. Whereas affect has an "explosive" dynamic and a short duration, the increase in mental tension can be relatively long, and the decline is not so rapid. The state of mental tension itself may also not be as short-lived as affect. Further, if affect unambiguously causes significant disorganization of mental activity, then, as noted above, mental tension may not only have a destructive effect on mental activity, but may also improve its quality, i.e. adaptation to negative influences is possible (it should be noted, however, that the adaptation syndrome is not limitless, and disorganization of mental activity will follow sooner or later).

As the main change of stress are changes in the functional level of activity, so it is manifested in the tension of this level, which causes its decrease and can contribute to the disorganization of activity in general. Involvement of intellectual processes and personality traits of an individual in the experience of adversely affecting stimuli on the body is conditioned by subjective features of human perception of stressogenic circumstances, which are the consequence of some uncharacteristic human behavior. And these harmful (stressogenic) circumstances for a person may be the circumstances of legal significance, when deciding an issue related to the implementation of the rights of a citizen.

According to V.L. Vasilyev's concept, it is impossible to allocate universal psychological stresses and universal situations causing psychological stress equally in all people. So, for example, even the weakest stimulus under certain conditions can play a role of psychological stress for a certain person, on whom another and even very strong stimulus has no influence. These circumstances are important in assessing the emotional state of a person during the period of interest to the civil court.

For those who, like Z. Freud, are inclined to explain mental manifestations by organic causes, anxiety is an extremely interesting problem because of its close connection with physiological processes

According to our understanding, anxiety is often accompanied by such physiological symptoms as: palpitations, perspiration, diarrhea, and rapid breathing. These physical signs appear in both lucid anxiety and unconscious anxiety. In our opinion, the aforementioned signs that determine anxiety have certain conditions that manifest themselves in emotional states responsible for the onset of anxiety status.

In our opinion, different individuals consider completely different things to be their vital values. State: acceptance of life, freedom, status of children - depends on what is the highest value for a person: body, property, situation, beliefs, work or love relationships?

As a primary link in the development of mental stress is frustration - a mental state that occurs when there is a blockage of meaningful needs, which is manifested in feelings of dissatisfaction and mental tension arising from the inability to realize certain goals.

So, the concept of frustration is definitely connected with the concept of needs, the range of unsatisfied needs can be very wide, as well as unconscious in its entirety. According to observations from our practical experience, the blockage of needs satisfaction is more often determined not by the quality of the outwardly manifested obstacle, but by the 
presence of competing needs in a person, i.e. by the internal conflict that leads a person to one or another degree of his reaction. From the above it follows that the probability of mental stress development at the increase of frustration tension depends on individualpsychological abilities of a person and the degree of stability of this person to the mental stress. In this case we are talking about "personal endurance", understood as a potential ability to actively overcome difficulties, which depends on the personal resources of a person who finds himself in these or those circumstances.

Also it is possible to note influence of emotional stress on certain behavioral features of the person, a special importance and role of previous experience and features of the personality which determine individual vulnerability.

The study of anxiety as a personal factor is the subject of the works of K. Horney [4]. According to the author, anxiety, like fear, is an emotional response to danger. Unlike fear, anxiety is characterized by vague and uncertainty. According to Goldstein, anxiety is caused by such a danger that threatens the very essence or core of the person. What explains the anxiety or helplessness of a person in the face of danger, or what is being threatened?

In the studies of F.B. Berezin formed views in favor of the "single nature" of anxiety. According to the author's position, all phenomenological manifestations of anxiety can be noted with the participation of the same hypothalamic structures and arise independently of premorbid personality traits, replacing each other when the severity of anxiety disorders changes.
According to the author, "anxiety-fearful excitement" is "the need for a motor discharge with a sense of the inevitability of impending disaster, the panic search for help manifests itself in anxiety-fearful excitement, which is the most pronounced of the disorders of the anxiety series. In anxiety-fearful agitation, the anxiety-induced disorganization of behavior reaches a maximum, and the possibility of purposeful activity disappears.

Accordingly, the anxiety series in the order of increasing severity includes the following phenomena: feelings of internal tensionhyperesthetic reactions-anxiety itself-fear-a sense of the inevitability of an impending catastrophe - anxiety-fearful excitement. With a paroxysmal (paroxysmal) increase in anxiety, all these phenomena can be observed during one paroxysm. In other cases, their change occurs gradually, and in relatively stable conditions, each of the elements of the alarm series is noted for a long time. As a rule, there is an inverse relationship between the stability of the state and the severity of anxiety disorders: the most stable states are those characterized by internal tension, the least stable are those whose picture is determined by a sense of the inevitability of an impending catastrophe and anxiety-fearful excitement.

The completeness of representation of elements of anxiety series depends on anxiety severity and intensity of its growth: at low intensity of anxiety its displays can be limited to a feeling of internal tension, at prompt growth of intensity the initial elements of a series can not be caught, at gradual development and sufficient intensity all elements of a series can be traced" [5].

According to the author's concept, anxiousfearful excitement is a "critical" manifestation 
of anxiety as such. The author also testifies that this factor is phenomenological for all types of individuals, regardless of their individual psychological properties.

Taking into account our research position, F. B. Berezin, representing the "anxious-fearful" stage of arousal, speaks of the culminating phase of the state experienced by the person who committed the crime in the state of affective tort.

In our opinion, the degree of experience of danger or threat (with an alarming component) is determined by the essence of the person himself, his individual psychological inclinations, as well as the genotype and the environment of education, which include: both the family atmosphere and the social environment.

Since our interest is devoted to the emotional states that determine a person's behavior in circumstances of crisis significance, whether it is a criminal incident or the circumstances of a civil lawsuit, it is necessary to consider such a concept as psychosomatics, and its influence on certain affective manifestations of a person. Psychosomatics (from the Greek psiho - "soul" and "soma" - "body") is [6]:

- A field that studies the interaction between behavior and somatic disease;

- Science, the objects of research of which are the soul and the body in their interaction.

Some authors (S. P. Botkin, M. Ya. Mudrov, G. A. Zakhar'in) have conducted studies of the dependence of the emotional state on health. They believed that the psychosomatic health of people is most affected by the personality itself, its emotional state, and not by biological factors. In turn, to make a correct somatic diagnosis, it is necessary to take into account the characteristics of the individual, the probability of which is determined by individual psychological properties.

The subject of our research is also the emotional states of the individual, whose somatic states in one way or another influenced the decision-making in legally significant circumstances of civil law.

Considering the strategies of personal behavior under stress, we will take as a basis the theory of coping with difficult life situations - the theory of coping.

Coping refers to the constantly changing cognitive behavioral attempts to cope with specific external and internal demands that are assessed as stress, or exceed the ability of a person to cope with them. Coping behavior is a form of behavior that reflects an individual's willingness to solve life problems, adapt to circumstances with the ability to use certain means to overcome emotional stress.

According to A. Maslow, the following methods of coping behavior are distinguished [7]: a) problem solving; c) search for social support; c) avoidance.

Coping behavior is realized through the use of various coping strategies based on the resources of the individual and the environment. One of the important environmental resources is social support. Personal resources include: positive selfesteem, low neuroticism, internal locus of control, optimistic outlook, empathic potential and other psychological constructs. Since coping processes are a part of the emotional response, the maintenance of emotional equilibrium through the reduction, elimination 
or removal of the acting stressor depends on them.

According to R. S. Lazarus (1991), at this stage, a secondary assessment of the latter is carried out. The result of the secondary assessment is one of three possible types of coping strategy:

a) Direct active actions of the individual in order to reduce or eliminate the danger (attack or flight, delight or love pleasure);

b) An indirect or mental form without direct influence, which is impossible due to internal or external inhibition, for example, displacement ("this does not concern me"), reassessment ("this is not so dangerous"), suppression, switching to another form of activity, changing the direction of the emotion in order to neutralize it, etc.;

c) Coping without emotions, when the threat to the person is not assessed as real (contact with means of transport, household appliances, everyday dangers that we successfully avoid).

Protective processes seek to rid the individual of the mismatch of motives and ambivalence of feelings, to protect him from the awareness of unwanted or painful emotions, and most importantly-to eliminate anxiety and tension. The effective maximum of protection is at the same time the minimum of what a successful coping is capable of. "Successful" coping behavior is described as: increasing the adaptive capabilities of the subject, its realism, flexibility, awareness, activity, including arbitrary choice.

In the context of our research, we will consider the concept of "unsuccessful coping" (in our terminology, anti - coping), which uses nonconstructive, but psychologically understandable (taking into account the personality and circumstances) strategies that deprive a person of the opportunity to make decisions taking into account all the necessary conditions.

According to our assumptions, there is a relationship between the personal constructs with which a person forms his attitude to life's difficulties and what strategy of behavior under stress (coping with the situation) he chooses.

According to the humanistic position of $\mathrm{E}$. Fromm, a person has the instinct of overcoming, one of the forms of manifestation of which is search activity, which ensures the participation of evolutionary and programmatic strategies in the interaction of the subject with various situations.

According to observations from our practical experience, the preferred ability and inability to cope are also influenced by individual psychological characteristics, including both temperament, level of anxiety, type of thinking, and orientation of character. The severity of certain ways of responding to difficult life situations is not only dependent on the self-actualization of the individual, but also depends on the degree of loss of this selfactualization. Also, according to the results of our empirical research (acts of forensic psychological examinations) in civil cases, the resolution of the situation of a complex legally significant period mostly depends on the degree of adequacy of the assessment of what is happening, and on the significance of the subjective assessment of the circumstances of the stressful period.

According to Scherrer, systematic errors in the assessment of the situation, which are 
observed in depressed individuals, can be defined by the term "evaluative pathology". A detailed diagnosis of the described condition can be of great importance both for the analysis of the conditions for the occurrence of the problem, and for the analysis of the legal consequences of "evaluative pathology" [1].

In the research aspect of E. D. Sokolova, F. B. Berezin, T. V. Barlas, the concepts of physiological and emotional stress are distinguished. According to the authors, physiological stress occurs in connection with direct physical impact, and emotional stress is caused by a complex stressful situation that affects a person through complex mental processes.

The subjective attitude to the stimulus, according to the authors, depends on: the unique individual experience, personal characteristics, the nature of cognitive processes-together determining the individual significance of the stressor [9].

Taking into account the topic of our research, the individual significance of the stressor is also fixed by a set of factors, including both age parameters and parameters of somatic health. The practical experience of our research allows us to express the opinion that no situation causes stress for all people without exception.

These statements are based on the meaning of a person's individual predisposition to adapt to a particular environmental impact, and moreover, to extreme circumstances perceived from the point of view of their subjective experiences. In the present context, we rely on the research criteria of experimental experience, determined by the position of the" individual predisposition " of the subject to experience events through the prism of subjective attitudes.

Hypothetical developments within the framework of research topics allow us to identify the following parameters:

a) Not every emotional impact leads to stress of the events experienced;

b) Not every stressful state leads to a state of frustrated tension;

c) Not every frustrated state leads to an affect that has (in behavioral terms) destructive consequences.

In our opinion, the center of gravity in assessing the emotional state, in particular, mental stress can not be transferred to the characteristics of the environment. In other words, stress is not a set of environmental influences, but an internal state of the individual (taking into account the state of the body), in which the implementation of the integrative functions of the body is complicated. It is also necessary to refer to "stressors", and their connections with mental deformities that have variable effects.

The research parameters determined by the framework of civil proceedings allow us to diagnose stressful states when making transactions of different nature, taking into account the psychological definition of deficit of volitional organization of behavior. Specificity of this diagnostics consists in qualitative characteristics defined by "stress" as a transition to "distress", essentially influencing behavioral parameters in view of acceptance of certain decisions.

In our opinion, a stress condition can both mobilize human resources and demobilize them. And if there is a question about influence 
of stress on behavioral features of the person in legally significant situations, it is necessary to reveal diagnostic criteria of "distress" state. Possibility of division of situations into normal, extreme and hyperextreme allows us to reveal in the person a level of extreme tension or overstrain. Extreme situations, in our opinion, are integral phenomena in the form of a temporary confluence of negative circumstances, expressed in special unfavorable conditions for human activity. An extreme situation presupposes that a subject is included in it. And this involvement can be objective (as complicated conditions), and subjective (attitudes, ways of action in sharply changing circumstances).

In our opinion, an extreme situation is a tense, complex and difficult situation, and therefore requires resolution. The consequences of such a situation, according to our assumptions, are both affective reactions leading to a violation of self-regulation, and to states of dissociation (disstress), leading to behavioral defectiveness, which determines the right to an untenable (with limited ability to act adequately) transaction of various complexity categories.

Thus, according to our research concept, a person who is in a state of hopelessness (taking into account the existing stressful circumstances, as well as taking into account his age and behavioral characteristics), suffers from a lack of cognitive abilities to make adequate decisions in accordance with the norm of law. And in this case, it is possible to diagnose a state of" distress " that leads a person to a defect in the volitional regulation of behavior that differs from the behavior that does not correspond to the legal characteristics of behavior in a frustrated state.

\section{REFERENCES}

1. Clinical psychology / Ed. by M. Perret, W. Baumann. - SPb., 2007. - P. 359.

2. Enikeev M. I. General and social psychology. - M., 1999. - P. 131.

3. Engalychev V. F., S. S. Shipsin Forensic psychological expertise in criminal and civil proceedings: questions of theory and practice. - M., 2015. - S. 122-177.

4. Horny K. Coll. Op. 3 t. - M., 1997. - P. 174180.

5. Berezin F. B. the Role of the hypothalamus in the mechanism of action of psychotropic drugs in the light of the study of psychopathology hypothalamic syndrome // Modern psychotropic drugs. - M., 1967. Issue 2. - p. 61-70.

6. Great psychological encyclopedia. - M., 2007. - P. 374.

7. Malkina-Pykh, I. Psychosomatics // the Handbook of a practical psychologist. - M., 2004. - S. 28.

8. Sokolova E. D. Berezin F. B., Barlas, T. V. Emotional stress: psychological mechanisms, clinical manifestations, and therapy. - M., 1996. -S. 5-25. 